\title{
Ultrasonic examination in severe diabetic eye disease
}

\author{
DAVID MCLEOD AND MARIE RESTORI \\ From the Department of Ultrasound, Moorfields Eye Hospital, City Road, London
}

SUMMARY B-scan ultrasound of the posterior segment is reported in 154 patients with severe diabetic eye disease. Epiretinal fibrosis, vitreous haemorrhage, vitreous detachment, and retinal detachment were frequently diagnosed. The ultrasonic findings are discussed in relation to the pathological changes in the vitreous in proliferative diabetic retinopathy.

Diabetic retinopathy is a common cause of blindness, visual loss resulting from macular oedema, vitreous haemorrhage, retinal detachment, rubeotic glaucoma, or major ocular vascular occlusion. Treatment of certain types of severe diabetic eye disease is now possible with the advent of new techniques of vitreous removal. In planning surgery ultrasonic evaluation is invaluable if the ocular media are opaque (Coleman, 1972; Jack et al., 1974), but no comprehensive report of the ultrasonic findings in diabetes has been published. In this paper we present our results in the last 154 consecutive diabetic patients examined in the Department of Ultrasound at Moorfields Eye Hospital.

\section{Materials and methods}

A rapid B-scan technique was used to examine 176 eyes of the last 154 patients referred for ultrasonic evaluation because of severe diabetic eye disease. Of these patients 63 were female and 121 were over 40 years of age.

The equipment used and the method of examination were described in previous publications (McLeod et al., 1977; Restori and McLeod, 1977), so only the most salient features are mentioned here. A $10 \mathrm{MHz}$ focused transducer was coupled to the anaesthetised eye by a saline bath, and a Barraquer speculum was used to give good global exposure. The B-scan level could be adjusted remotely by 2 buttons close to the display tube. B-scan sections were normally observed in the horizontal plane at $1 \mathrm{~mm}$ intervals with all directions of gaze. Dynamic studies were performed by observing movements of abnormalities on the B-scan during and following a change in direction of gaze. Good grey scale on the B-scan display

Correspondence to Mr D. McLeod, Moorfields Eye Hospital, City Road, London EC1V 2PD.
Table 1 Prevalence of ultrasonic abnormalities in 176 eyes

\begin{tabular}{|c|c|c|}
\hline $\begin{array}{l}\text { Vitreous } \\
\text { haemorrhage }\end{array}$ & $\begin{array}{l}\text { Intragel echoes } \\
\text { Retrohyaloid echoes } \\
\text { Both intragel and retrohyaloid } \\
\text { Not detected }\end{array}$ & $\begin{array}{r}101 / 176(57 \%) \\
86 / 176(49 \%) \\
53 / 176(30 \%) \\
42 / 176(24 \%)\end{array}$ \\
\hline $\begin{array}{l}\text { Epiretinal } \\
\text { fibrosis }\end{array}$ & Detected & $74 / 176(42 \%)$ \\
\hline \multirow[t]{4}{*}{$\begin{array}{l}\text { Vitreous } \\
\text { detachment }\end{array}$} & $\begin{array}{l}\text { Diagnosed by compartment } \\
\text { echoes } \\
\text { Diagnosed by interface echoes } \\
\text { Both compartment and interface } \\
\text { Not detected }\end{array}$ & $\begin{array}{c}107 / 176(61 \%) \\
149 / 176(85 \%) \\
89 / 176(51 \%) \\
9 / 176(5 \%)\end{array}$ \\
\hline & $\begin{array}{l}\text { Predominantly rigid interface } \\
\text { Predominantly mobile interface } \\
\text { Compaction sign (ochre } \\
\text { membrane) }\end{array}$ & $\begin{array}{l}91 / 167(55 \%) \\
64 / 167(38 \%) \\
12 / 167(7 \%)\end{array}$ \\
\hline & $\begin{array}{l}\text { Complete vitreous detachment } \\
\text { Incomplete vitreous detachment }\end{array}$ & $\begin{array}{r}17 / 167(10 \%) \\
150 / 167(90 \%)\end{array}$ \\
\hline & $\begin{array}{l}\text { Localised vitreoretinal adhesion } \\
\text { Extensive vitreoretinal adhesion } \\
\text { Multiple vitreoretinal adhesions } \\
\text { Partial detachment/unclassified }\end{array}$ & $\begin{array}{l}40 / 150(27 \%) \\
72 / 150(48 \%) \\
22 / 150(15 \%) \\
16 / 150(10 \%)\end{array}$ \\
\hline $\begin{array}{l}\text { Retinal } \\
\text { detachment }\end{array}$ & $\begin{array}{l}\text { Detected } \\
\text { Localised detachment } \\
\text { Subtotal/total detachment } \\
\text { Subretinal echoes }\end{array}$ & $\begin{array}{r}100 / 176(57 \%) \\
82 / 100(82 \%) \\
18 / 100(18 \%) \\
10 / 100(10 \%)\end{array}$ \\
\hline
\end{tabular}

obviated the need for A-scan studies in most cases. The tonal quality of the Polaroid prints selected for publication is not representative of that observed directly on the display screen.

\section{Results}

Vitreous haemorrhage, epiretinal fibrosis, posterior vitreous detachment, and retinal detachment could be diagnosed by ultrasonic examination. Table 1 presents the prevalance of these abnormalities in our series.

Haemorrhage within the vitreous cavity gave rise to scattered point-like echoes of varying amplitudes (Figs. 1a, b, c). Arrangement of such echoes 
to form intragel membranes was sometimes observed (Figs. 1d, e), while sedimentation of haemorrhage within fluid vitreous produced a flat sheet ('fluidlevel') of very high amplitude echoes (Figs. 1f, g). If a sheet of high amplitude echoes lower in amplitude than sedimented blood but showing more internal structure was detected on the retinal surface, the echoes were considered to arise from epiretinal fibrous tissue (Fig. 1h).

Posterior vitreous detachment was sometimes indicated by the presence of point-like echoes confined to the gel compartment or retrohyaloid space (Figs. 1a, b, d, i, j). The distinction between these compartments was often emphasised during
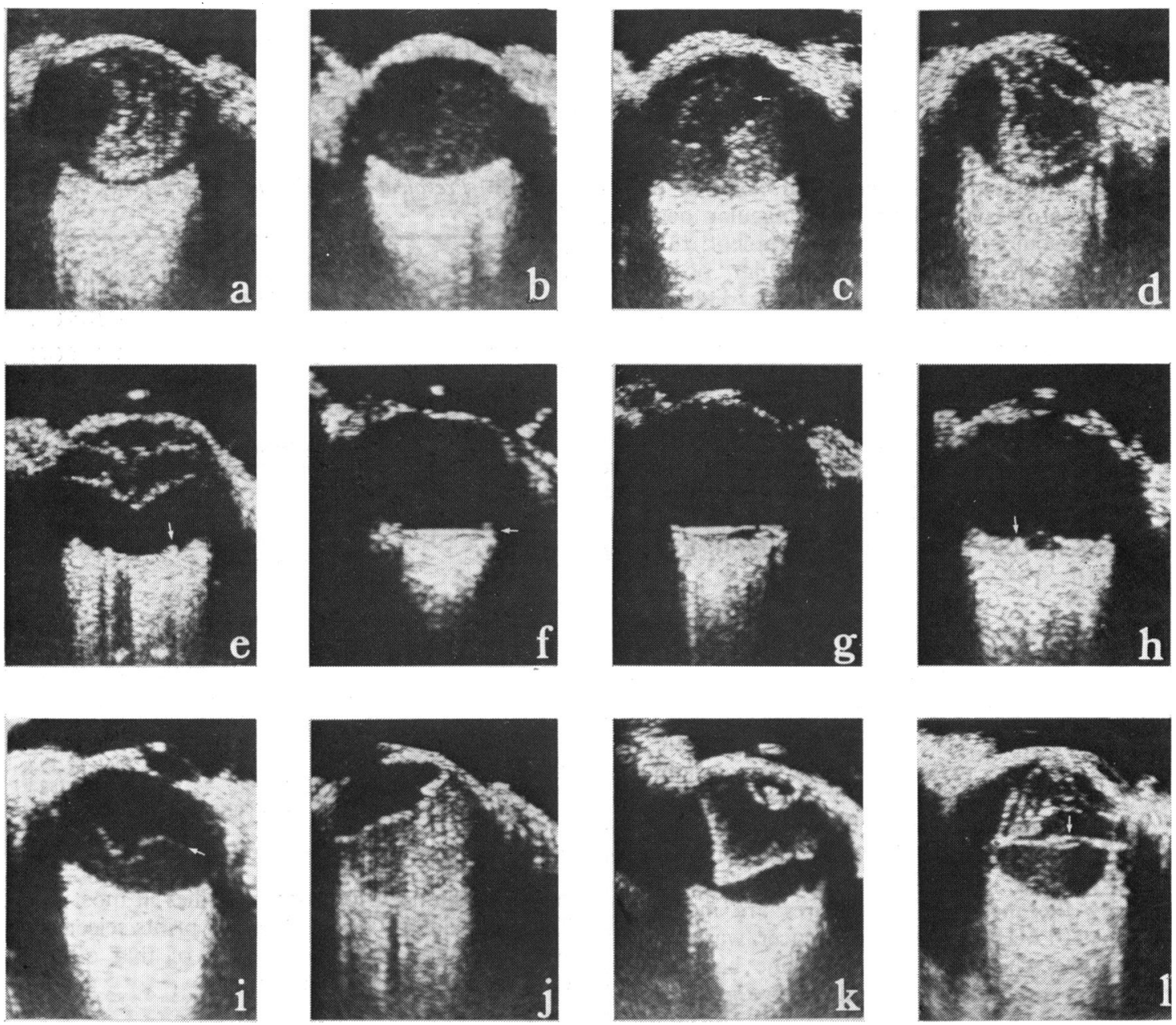

Fig. 1 Horizontal B-scan sections. (a) Haemorrhage in mobile detached gel; clear retrohyaloid space.

(b) Retrohyaloid haemorrhage; clear gel. (c) Retrohyaloid haemorrhage; central gel haemorrhage with clear cortical gel (arrow). (d) Eye deviated to right; intragel haemorrhage with some arrangement into membranes. (e) Intragel haemorrhage arranged into anterior intragel and posterior hyaloid membranes; clear retrohyaloid space; lens related artefact (Baum's bumps) on posterior retina (arrow): (f) 'Fluid-level' from sedimentation of blood in fluid vitreous (arrow). (g) Eye deviated to right; 'fluid-level' of sedimented blood. (h) Thick fibrovascular epiretinal membranes on posterior retina (arrow) excluding the macula. (i) Retrohyaloid haemorrhage and mobile posterior hyaloid membrane (arrow); clear gel. (j) Eye deviated to left; dense retrohyaloid haemorrhage and posterior hyaloid membrane outlined; clear retracted gel. $(\mathrm{k})$ Eye deviated to right; intragel haemorrhage compacting in cortical gel to form 'ochre membrane'; gel tethered to optic disc. (1) Eye deviated to right; fibrotic posterior hyaloid membrane (arrow) between vitreous base anteriorly and posterior vitreoretinal adhesion; central intragel and retrohyaloid haemorrhage. 

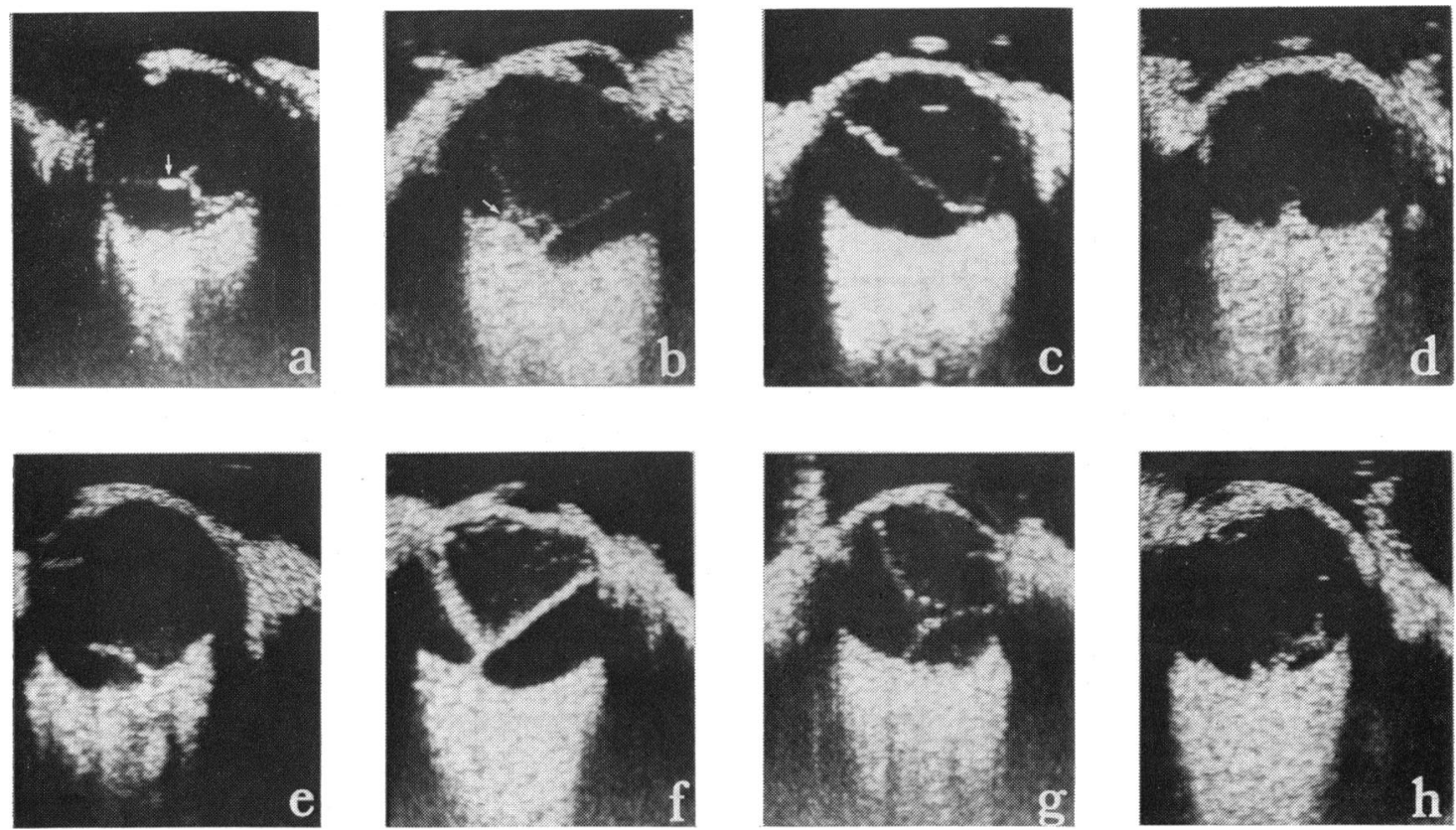

Fig. 2 Horizontal B-scan sections. (a) Eye deviated to left; fibrovascular stalk from optic disc; fibrocellular and fibrovascular (arrow) components of rigid posterior hyaloid membrane; traction retinal detachment at base of peripapillary stalk. (b) Eye deviated to right; posterior hyaloid membrane between vitreous base anteriorly and margins of extensive vitreoretinal adhesion in posterior pole (arrow). (c) Incomplete posterior vitreous detachment with temporal vitreoretinal adhesion. (d) Thick fibrotic stalk from optic disc. (e) Rigid prepapillary fibrovascular stalk and retrohyaloid haemorrhage. (f) Intragel haemorrhage with arrangement along posterior hyaloid interface; stalk-like vitreoretinal adhesion posteriorly. (g) Eye deviated to right; detached gel outlined by haemorrhage, and prepapillary vitreoretinal adhesion. (h) Eye deviated to left; 2 posterior vitreoretinal adhesions.

dynamic studies, the detached gel moving as a corporate body within the fluid retrohyaloid space. Alternatively, or additionally, posterior vitreous detachment was indicated by the presence of echoes from the posterior hyaloid interface. If the gel was mobile and contained opacity, echoes along this interface were considered to arise from blood products in the cortical gel (Figs. 1d, e); a gradual compaction of such intragel echoes within the posterior hyaloid interface was characteristic of 'ochre membrane' formation (Fig. 1k). If a fine sheet of high amplitude echoes arose from the posterior hyaloid interface but the gel boundary was rigid, the posterior hyaloid membrane was considered to be fibrocellular in nature (Fig. 11). In other cases a thicker sheet of high amplitude echoes arose from the rigid gel boundary, and the posterior hyaloid membrane was considered to be composed of fibrovascular tissue (Fig. 2a). On occasions, several different types of membrane were found in the same eye.

Posterior vitreous detachment could be complete or incomplete. If incomplete, there might only be a single vitreoretinal adhesion (Figs. 2a, b, c), which was often stalk-like (Figs. 2d, e, f, g). Multiple vitreoretinal adhesions were also demonstrable (Fig. 2h), especially by consecutive serial scanning of various levels within the eye.

Detachment of the retina was indicated by membrane-like echoes of high amplitude which tethered at the ora serrata and the optic nerve head when total. Some retinal detachments showed undulating movements on dynamic testing, but movement of the retina was usually restricted or absent. Occasionally a fibrotic posterior hyaloid membrane tethering at the optic nerve head mimicked a total retinal detachment in topography and in echo amplitude (Fig. 3a).

In this series, however, retinal detachment was frequently localised, for example, traction detachment at the base of a vitreoretinal stalk (Figs. 2a and $3 \mathrm{~b}$ ); more extensive detachments were related to wider areas (or multiple areas) of vitreoretinal adhesion (Figs. 3c, d, e, f, g, h). In other eyes an anterior detachment of the retina was noted in the vitreous base region (Figs. 3i, j) with a rigid hyaloid 
membrane connecting the detached basal retina to posterior sites of vitreoretinal adhesion. In several cases point-like echoes were detected in the subretinal space, particularly when the history suggested a long-standing retinal detachment (Figs. $3 \mathrm{k}, \mathrm{l})$.
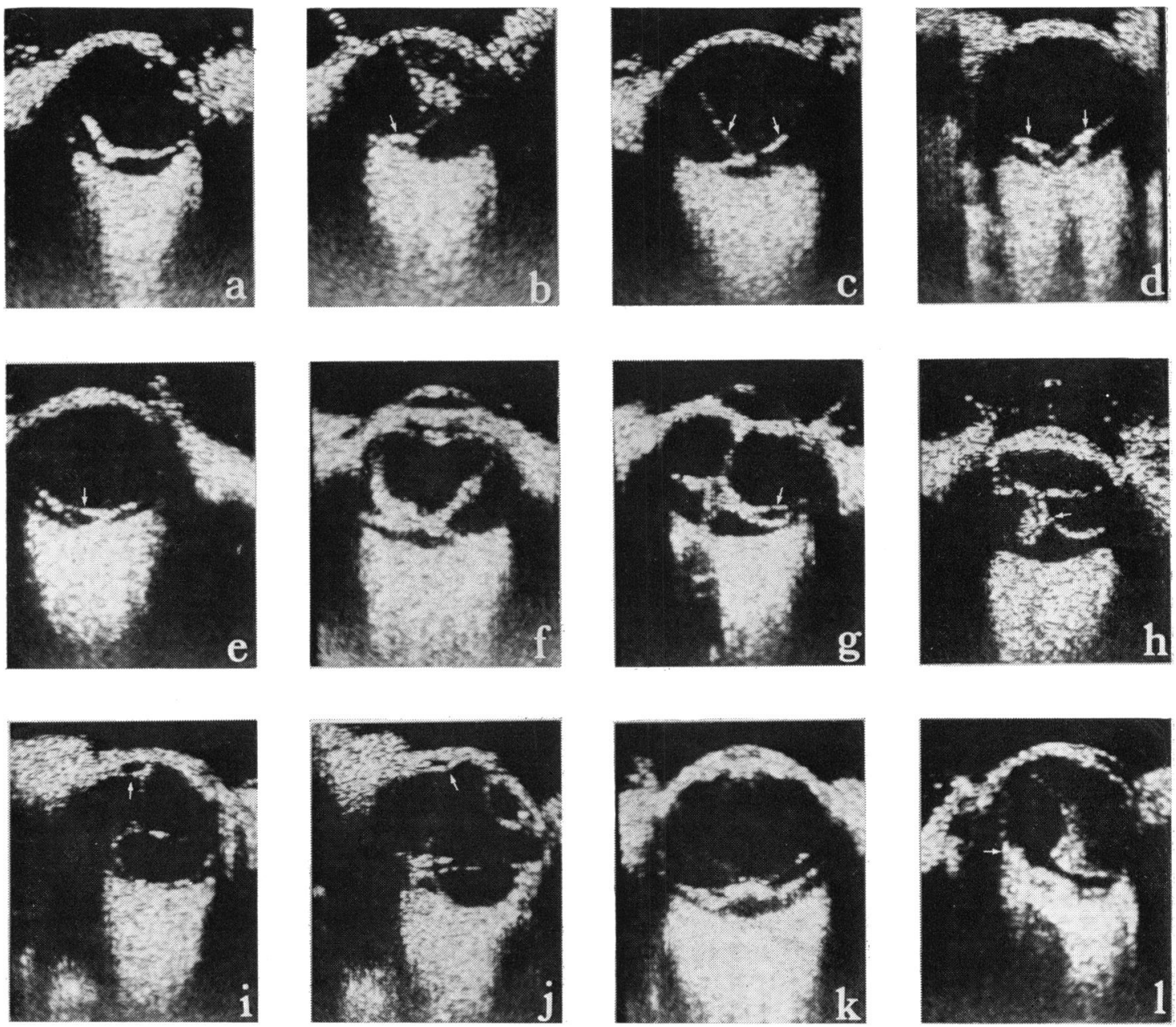

Fig. 3 B-scan sections. (a) Eye deviated to right; gross fibrovascular proliferation tethering at disc. (b) Eye deviated to right; haemorrhage in collapsed rigid gel; localised retinal detachment around base of vitreoretinal adhesion (arrow). (c) Localised detachment of posterior retina; posterior hyaloid membrane (arrows) inserts into epiretinal fibrous tissue at summit of traction. (d) Vertical B-scan section; retinal detachment tethering at optic disc; fibrovascular epiretinal tissue at summit of traction (arrows); fibrocellular posterior hyaloid membrane bridging between posterior vitreoretinal adhesions. (e) Two localised shallow retinal detachments and 'bridging' posterior hyaloid membrane (arrow). (f) Extensive fibrotic vitreoretinal adhesion and traction retinal detachment. (g) Extensively detached retina tethering at optic disc; widespread vitreoretinal adhesions and rigid posterior hyaloid membrane (arrow). (h) Grossly detached retina (arrow) suspended from rigid retracted posterior hyaloid membrane. (i) Eye deviated to right; posterior hyaloid membrane and stalk; traction retinal detachment in vitreous base region (arrow). (j) Eye deviated to right; retinal detachment at vitreous base anteriorly (arrow) and in posterior pole. (k) Extensive fibrotic traction retinal detachment and subretinal echoes. (l) Eye deviated to right; haemorrhage in detached gel; clear retrohyaloid space; shallowly detached retina and echoes from subretinal space (arrow). 
lamina of the retina and grow within the cortical gel to form vascularised epiretinal membranes. The presence of extensive fibrovascular membranes on the retinal surface may be detected by ultrasound, since the normal smooth concave contour of the posterior globe is disrupted. Nevertheless, care must be taken to differentiate such fibrosis from shallow retinal detachment, a fluid level of dense haemorrhage on the retinal surface, and artefacts produced by the lens (for example, Baum's bumps).

There are 3 important consequences of diabetic epiretinal vasoproliferation: (i) fibrous contraction within the epiretinal membrane and consequent tangential traction on the retina; (ii) an exaggerated adhesion between the vitreous gel and the retina owing to incarceration of cortical gel in the vascularised epiretinal membrane; (iii) separation of the cortical gel from the retinal except at these sites of exaggerated adhesion (and also excepting the adhesion at the vitreous base)_'incomplete posterior vitreous detachment'.

During or after vitreous detachment bleeding often occurs into the vitreous cavity owing to traction on new vessels at sites of persistent vitreoretinal adhesion (Davis, 1965; Tolentino et al., 1966). Haemorrhage may be located within the gel, in the retrohyaloid space, or in both compartments, and is the commonest indication for ultrasonic examination in diabetic eye disease. In those eyes in which the detached gel retains its mobility an apparent 'compaction' of blood on the inner aspect of the posterior hyaloid interface can sometimes be observed-'ochre membrane formation'. However, fully formed ochre membranes proved to be relatively infrequent in diabetic vitreous haemorrhage (Table 1).

The detached posterior hyaloid interface often forms a scaffold on which fibrovascular tissue proliferates and extends anteriorly-'forward new vessels' or 'retinitis proliferans'. In addition the more anterior part of the posterior hyaloid interface may also be immobilised by condensation of the cortical gel or proliferation of a non-vascularised fibrocellular membrane connecting the posterior border of the vitreous base to the retinitis proliferans (Lee, 1968). The fibrocellular and fibrovascular components of the posterior hyaloid membrane can often be delineated and differentiated by ultrasound, fibrovascular tissue giving rise to a series of echoes of longer duration than fibrocellular membranes.

In some eyes in this series both mobile and rigid components of the posterior hyaloid interface could be detected. In general, however, echo compaction and ochre membrane formation (characterised by gel mobility) and fibrotic membrane formation (which is characteristically rigid) were mutually exclusive. Fibrovascular invasion (or 'organisation') did not involve the gel itself in our cases; the apparent 'vascular pulsation' detected by A-scan techniques (Ossoinig, 1972) in so-called 'organised' gel probably arose from mobile intragel opacity (McLeod and Restori, 1977).

Contraction of fibrous tissue on the retinal surface (tangential traction) and along the detached posterior hyaloid interface (anteroposterior and bridging traction) may ultimately result in retinal detachment (as in over half of the eyes in this series). Diabetic traction detachment has a characteristic ultrasonic appearance: the retinal elevation is angular in configuration and immobile on dynamic testing; there is associated epiretinal fibrosis at the summit of traction to which the posterior hyaloid membrane is attached; and the retinal surface has an anterior concavity between the limit of detachment and the vitreoretinal adhesion. However, in a majority of extensive diabetic detachments there are associated retinal holes (Tasman, 1972), so the detached retina may have an anterior convexity, and dynamic testing may even reveal a degree of retinal mobility.

Posterior detachment of the vitreous is a crucial stage in the progression of proliferative retinopathy to haemorrhage and retinal detachment (Davis, 1965). In our series vitreous detachment was identified ultrasonically in $95 \%$ of eyes. An ultrasonic diagnosis of 'complete posterior vitreous detachment' was made in $10 \%$ of these eyes. This was probably an overestimate, since peripheral postbasal vitreoretinal adhesions are often difficult to detect, especially in the presence of retrohyaloid haemorrhage. Nevertheless in some elderly patients a highly mobile gel was identified in the anterior part of the vitreous cavity - the typical ultrasonic pattern of 'posterior vitreous detachment with collapse'.

The more usual incomplete posterior vitreous detachment of proliferative retinopathy results from variable numbers and sites of persistent vitreoretinal adhesion (Foos, 1972). Such adhesions usually indicate, by inference, the extent of epiretinal fibrovascular proliferation present at the time of vitreous detachment. In some eyes neovascularisation is very limited in extent, the detached gel being tethered posteriorly only by a single adhesion to the optic nerve head. Retinal detachment is not generally seen in such cases, except occasionally round the base of the prepapillary stalk. In many eyes, however, fibrovascular proliferation on the surface of the retina is extensive by the time of posterior vitreous detachment: this results in multiple or widespread sites of persistent vitreoretinal adhesion. It is in these eyes that associated retinal 
detachment is generally observed, and crucial clinical decisions on vitreous surgery often depend on the ultrasonic diagnosis of retinal detachment, especially if the macular retina is involved. However, those eyes with extreme degrees of fibrotic rigidity of the retina or dense subretinal opacity are generally deemed inoperable.

We thank Miss Penny Davies for typing the manuscript.

\section{References}

Coleman, D. J. (1972). Ultrasound in vitreous surgery. Transactions of the American Academy of Ophthalmology and Otolaryngology, 76, 467-479.

Davis, M. D. (1965). Vitreous contraction in proliferative diabetic retinopathy. Archives of Ophthalmology, 74, 741-751.

Foos, R. Y. (1972). Posterior vitreous detachment. Transactions of the American Academy of Ophthalmology and Otolaryngology, 76, 480-496.
Jack, R. L., Hutton, W. L., and Machemer, R. (1974). Ultrasonography and vitrectomy. American Journal of Ophthalmology, 78, 265-274.

Lee, P. F. (1968). Clinical and histological findings in diabetic retinopathy. American Journal of Ophthalmology, 65, 688-695.

McLeod, D., Restori, M., and Wright, J. E. (1977). Rapid B-scanning of the vitreous. British Journal of Ophthalmology, 61, 437-445.

McLeod, D., and Restori, M. (1977). Real-time B-scanning of the vitreous. Transactions of the Ophthalmological Societies of the United Kingdom, 97, 547-550.

Ossoinig, K. C. (1972). Clinical echo-ophthalmography. Current Concepts of Ophthalmology, 3, 101-130.

Restori, M., and McLeod, D. (1977). Ultrasound in previtrectomy assessment. Transactions of the Ophthalmological Societies of the United Kingdom, 97, 232-234.

Tasman, W. (1972). Retinal detachment secondary to proliferative diabetic retinopathy. Archives of Ophthalmology, 87, 286-289.

Tolentino, F. I., Lee, P. F., and Schepens, C. L. (1966). Biomiscroscopic study of vitreous cavity in diabetic retinopathy. Archives of Ophthalmology, 75, 238-246. 